\title{
Theoretical Simulation for Evaluating Error in Irradiance Measurement Using Optical Detectors Having Different Cosine Responses
}

\author{
Vijeta $^{1,2}$, R. K. Kapri ${ }^{2}$, S. Saha ${ }^{2}$, V. K. Jaiswal ${ }^{2}$ and P. Sharma ${ }^{1,2} *$ \\ ${ }^{1}$ Academy of Scientific and Innovative Research (AcSIR), Ghaziabad 201002, India \\ ${ }^{2}$ Optical Radiation Metrology Section, CSIR-National Physical Laboratory, Dr. K.S. Krishnan Marg, New Delhi \\ 110012, India
}

Received: 11 March 2021 / Accepted: 09 June 2021 / Published online: 27 June 2021

(C) Metrology Society of India 2021

\begin{abstract}
Cosine response is an important characteristic of an optical detector for irradiance measurements. The non-ideal cosine response of a detector may lead to errors in irradiance measurements. In this paper, a theoretical simulation of irradiance generated due to isotropic point light sources is carried out for different illumination conditions. Simulation results show that the errors in irradiance measurements due to the cosine error of detectors become significantly high at the edges and corners of the floor when a light source is placed at the center of the roof. Further, it is observed that the errors are more in the enclosures having a height smaller than the floor dimensions. Even calibrated detectors measure erratic values of irradiance for a wider angle of incidence. Therefore, cosine errors in irradiance measurements are of great importance especially during the current scenarios of the COVID-19 pandemic, for ensuring the correct dose of Ultraviolet Germicidal Irradiation (UVGI).
\end{abstract}

Keywords: Irradiance; Calibration; Cosine error; Simulation

\section{Introduction}

With the evolution of humanity, artificial lighting has become an integral part of life [1]. Consequently, its measurement techniques have also started gaining importance [2, 3]. Artificial lighting is not only restricted to provide visual stimulation but is being used in a plethora of applications ranging from medicine, surgery, germicidal use, communication, and in industries [4-6]. Light, being at the helm of social, commercial, intellectual, and scientific application, requires reliable measurements [3].

Light measurement can be broadly categorized into radiometry and photometry. On one hand, radiometry relates to the measurement of the optical power of illumination while photometry is about the measurement of light as perceived by the human eye [3, 7]. Measurement techniques in photometry and radiometry are similar with a difference that the former is a physiological aspect while the latter is the physical aspect of light measurements $[7,8]$. However, physiological photometry can be

*Corresponding author, E-mail: sharmap2@nplindia.org converted into physical photometry by radiometric measurements weighted by the human eye response.

Radiometric and photometric measurements have gained much importance with the advancement in various applications of artificial light sources especially in the current pandemic scenario $[9,10]$. In the present situation of the COVID-19 pandemic, the use of the ultraviolet spectrum for germicidal irradiation has increased manifold. The UVC radiation $(100 \mathrm{~nm}-280 \mathrm{~nm})$, in the ultraviolet spectrum, exhibits enhanced germicidal efficacy and impedes the spread of infection through incapacitating microorganisms and viruses [5, 10]. However, quantification of UV dose is quite important for effective disinfection [11-13]. The UV dose depends on exposure time [14, 15] and irradiation geometry [16]. Hence, it is important to study and measure the irradiation patterns of the UV sources accurately. Apart from UV sources, the measurement of irradiance finds importance in illumination, therapeutic applications, etc.

Irradiance can be measured quantitatively by employing an optical detector. Optical detectors are the devices, when exposed to optical radiation, generate an electrical signal. The generated electrical signal is proportional to the radiant 
flux incident on the detector surface. This radiant flux falling on surface of unit area is called irradiance in the context of radiometry $[17,18]$. Thus, a detector signal is directly proportional to irradiance [19]. The photometric parameter corresponding to irradiance is illuminance. Different types of detectors are used in radiometry and photometry. Before measurement of irradiance or illuminance, calibration of the detector is very necessary. Detectors are calibrated for their spectral power responsivity using various methods having traceability to primary standards [20]. These calibrations are done in the laboratory under ideal conditions in which detectors are aligned in line horizontally to have a normal incidence from the source. However, the practical measurements are not always in a condition as conducive as that of the laboratory. In such cases, the detectors should have a good cosine response [21, 22].

A detector having an ideal cosine response shows a decline in measured irradiance by a factor of $\cos \theta$ as the illumination angle deviates from normal incidence by an angle $\theta$. This property is known as the cosine law [23, 24].

In the present manuscript, an attempt has been made to highlight the importance of cosine correction for irradiance measurements. This study has been carried out through theoretical simulation of light measurements in a cubical room. The model can further be used for the optimization of the position of light sources for proper illumination at different places like laboratories, industries, hospitals, and schools.

\section{Mathematical Modeling}

A mathematical model is prepared and theoretical simulations are conducted considering the practical illumination scenarios like illuminance measurements inside a closed room. For this, we have considered a cubical enclosure with different lighting conditions at the top face. All the light sources are considered to be isotropic point sources, and reflections from walls are also neglected.

\subsection{Source at the Center of the Roof}

The isotropic point light source is the basis of the simplest model for light measurements [12, 13]. Also, radiant intensity and irradiance are two important parameters for a light source application. The radiant intensity $(I)$ is the radiant power $(\mathrm{d} P)$ leaving the source in a certain direction through the unit solid angle made in that direction [13],

$I=\frac{\mathrm{d} P}{\mathrm{~d} \omega}$ where $\mathrm{d} \omega$ is the differential solid angle of the elemental cone in the given direction.

The irradiance $(E)$ is defined as the radiant power/flux incident on unit area of detector surface [13],

$E=\frac{\mathrm{d} P}{\mathrm{~d} A}$

$E=\frac{\mathrm{d} P}{\mathrm{~d} \omega r^{2}}=\frac{\mathrm{I}}{r^{2}}$

where the differential surface area $\mathrm{d} A$ is on the surface of a sphere centered on and at a distance $r$ from the source, and $E$ is the irradiance at that surface.

In the present case, the isotropic point light source ' $S$ ', shown in Fig. 1, is assumed to be placed at the center of the top face of the cubical enclosure. Then irradiance is measured by an optical detector (facing toward the roof), considered to be placed at an arbitrary position on the lower surface of the enclosure.

The situation for modeling in Fig. 1, where the detector experiences only normal incidence (at point $\mathrm{N}$ having source-detector distance of $h_{0}$ ) and an arbitrary point where

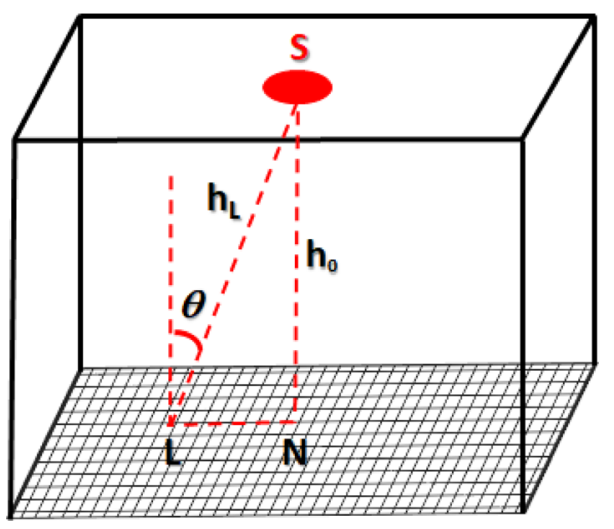

Fig. 1 Schematic diagram of a cubical enclosure having single light source
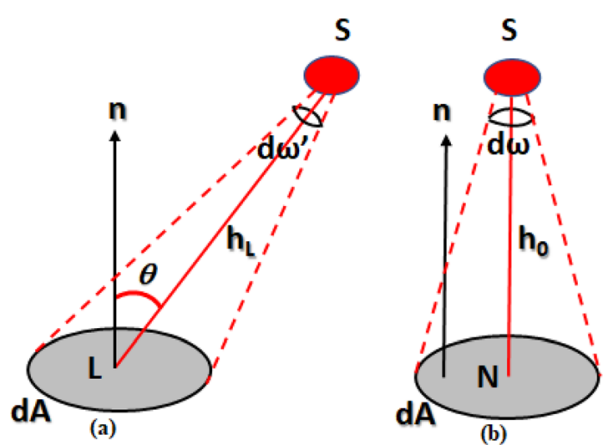

Fig. 2 Detector of area dA at different positions: a at distance $h_{L}$ from point source $\mathrm{S}$ at point $\mathrm{L}, \mathbf{b}$ at distance $h_{0}$ from point source $\mathrm{S}$ at point $\mathrm{N}$ 
the detector experiences an oblique incidence (at point $\mathrm{L}$ having source-detector distance of $h_{\mathrm{L}}$ ), is shown in Fig. 2.

From the definition of solid angle, we can write solid angle for case a and b (Fig. 2) as,

$d \omega^{\prime}=\frac{d A \cos \theta}{h_{L}^{2}}$

$\mathrm{d} \omega=\frac{\mathrm{d} A}{h_{0}^{2}}$

Then using Eqs. 1, 2, 4, and 5, the irradiance at point $\mathrm{L}$ and $\mathrm{N}$ can be written as,

$E_{\mathrm{L}}=\frac{\mathrm{I} \cos \theta}{h_{\mathrm{L}}^{2}}$

$E_{\mathrm{N}}=\frac{\mathrm{I}}{h_{0}^{2}}$

where $I$ is the intensity of the source in the given direction, and $h_{\mathrm{L}}$ and $h_{0}$ are the distances from the source to the surface element $\mathrm{d} A$.

It can be seen from Eq. 6 that irradiance from a point source has both inverse square law relationship [13, 25] and cosine dependence [13, 23]. Since radiant intensity remains same in all directions, hence Eqs. 6 and 7 may be combined as,

$\frac{E_{\mathrm{L}}}{E_{\mathrm{N}}}=\left(\frac{h_{0}^{2}}{h_{\mathrm{L}}^{2}}\right) \cos \theta$

From Fig. 1, we can write that $\frac{h_{0}{ }^{2}}{h_{\mathrm{L}}{ }^{2}}=\cos ^{2} \theta$. Thus,

$E_{\mathrm{L}}=E_{\mathrm{N}} \cos ^{2} \theta \times \cos \theta$

The above expression represents the resulting irradiance measured by a detector following an ideal cosine response at an arbitrary grid point.

Four detectors (A, B, C, and D) having non-ideal cosine responses have been considered and are used to evaluate irradiance theoretically at each grid point.

The integrated cosine errors ( $f_{2}$ values) of Detectors A, $\mathrm{B}, \mathrm{C}$, and $\mathrm{D}$ for $\theta$ in the range $0^{\circ}$ to $85^{\circ}$ interval are 25.02 , 42.24, 47.32, and 51.66, respectively [21]. The cosine responses of these four detectors along with ideal cosine response are shown in Fig. 3.

It is important to note that the calculations have been done by taking maximum irradiance $\left(1 \mathrm{~W} / \mathrm{m}^{2}\right)$ corresponding to the normal incidence position. The cosine responses have been theoretically simulated based on the response of some commercially available detectors. It may be observed that out of the four detectors, Detector A has cosine response closest to the ideal while Detector D has the worst. Then the irradiance at point $\mathrm{L}$, measured by a detector having non- ideal cosine response is given as,

$E_{\mathrm{L}^{\prime}}=E_{\mathrm{N}} \cos ^{2} \theta \times f(\theta)$

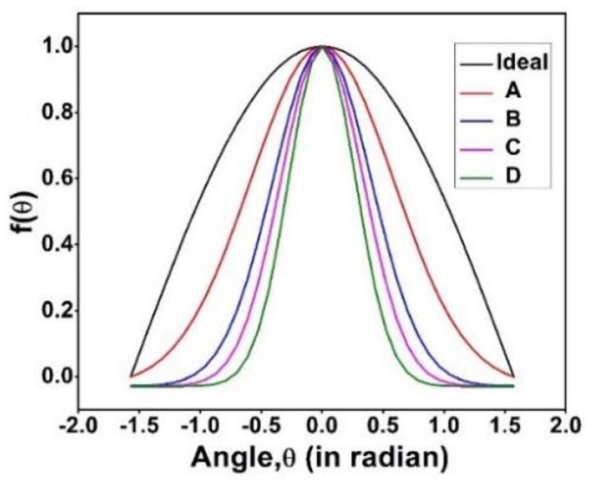

Fig. 3 Cosine response of detectors A, B, C, and D

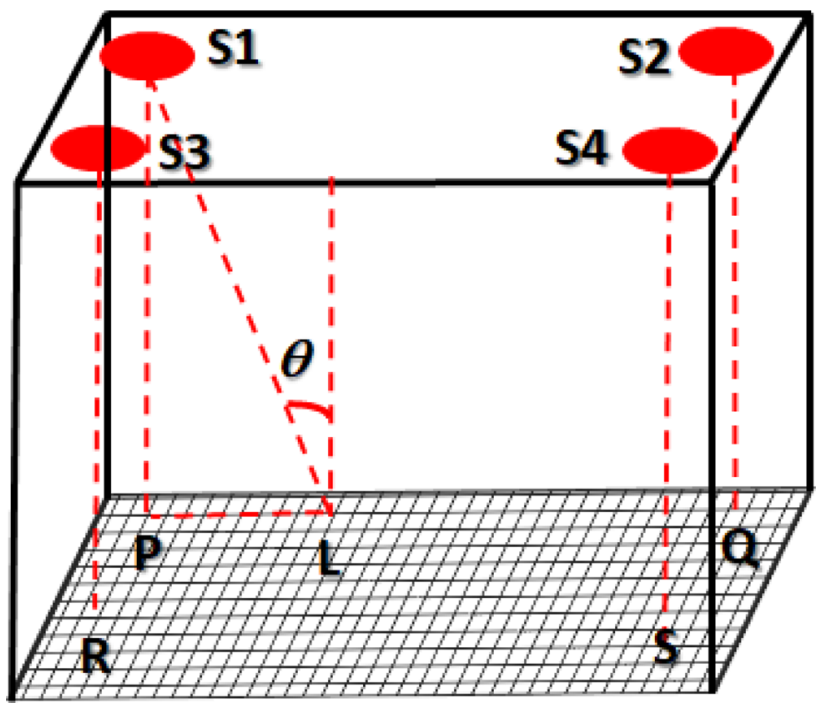

Fig. 4 Schematic diagram of the four light sources placed at the corners in a cubical enclosure

where $E_{L^{\prime}}$ is the irradiance measured by the detector having a non-ideal cosine response mathematically given by a $\theta$ dependent function $f(\theta)$.

Similarly, the irradiance at each of the grid points of the floor is calculated using Eq. 10.

\subsection{Source at four corners of the roof}

To understand the impact of multiple sources in an enclosure, four isotropic point light sources S1, S2, S3, and $\mathrm{S} 4$ are assumed to be placed at the corners of the top face (roof) of the cubical enclosure (Fig. 4). The irradiance just below four light sources S1, S2, S3, and S4 (normal incidence point) is assumed to be $1 \mathrm{~W} / \mathrm{m}^{2}$.

Then irradiance due to these light sources is calculated, considering optical detector following ideal and non-ideal cosine response at an arbitrary point on the floor of the room, using Eqs. 9 and 10 independently. The total 
irradiance measured by any detector at a point $\mathrm{L}$ would be the sum of irradiance due to light sources S1, S2, S3, and S4 individually.

\subsection{Source at the Center and Four Corners of the Roof}

Five isotropic point light sources (four at the corners and one at the center) are considered to be placed at the top face of the cubical enclosure as shown in Fig. 5. The irradiance at the normal incidence positions (P, Q, R, S, and N) of the individual light sources is imagined to be $1 \mathrm{~W} / \mathrm{m}^{2}$. Further, the resultant irradiance measured by any detector at all grid points on the lower surface is calculated using Eq. 10. This irradiance is the sum of irradiance of sources S1, S2, S3, S4, and S.

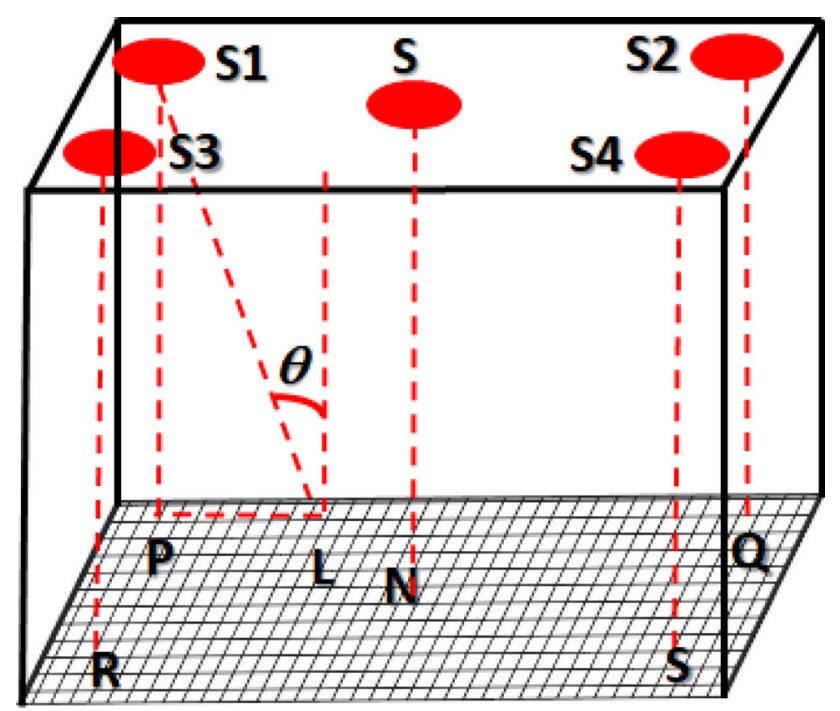

Fig. 5 Schematic diagram of the four light sources placed at the corners and one at the center in a cubical enclosure

\subsection{Error in Irradiance Measurement}

The relative error in irradiance measured by the detectors having poor cosine responses to the detector having ideal cosine response is calculated at each of the grid points of the floor, using the formula:

Error $=\frac{\left[E_{L}-E_{L^{\prime}}\right]}{E_{L}}$

\section{Results and Discussion}

Under different lighting conditions, irradiance profile measured by a detector following the true cosine response is shown by the contour plots in Fig. 6 (simulations have been done using Eq. 9). The height of the enclosure has been considered to be $3 \mathrm{~m}$. On assuming maximum irradiance just below the light source as $1 \mathrm{~W} / \mathrm{m}^{2}$, it can be seen that the irradiance falls to $0.54 \mathrm{~W} / \mathrm{m}^{2}$ (Fig. 6a) at the corners of the floor of the room when the source is placed at the center of the roof. On placing four isotropic point light sources at the corners of the roof, the irradiance measured at the center of the enclosure is $2.17 \mathrm{~W} / \mathrm{m}^{2}$ while that at the corners is $1.89 \mathrm{~W} / \mathrm{m}^{2}$ (Fig. 6b). Then simulations are done for the four light sources at corners along with one at the center of the top face. It can be seen that irradiance measured at the center of the floor of the enclosure to be $3.17 \mathrm{~W} / \mathrm{m}^{2}$ and $2.44 \mathrm{~W} / \mathrm{m}^{2}$ at the corners (Fig. 6c) of the floor of the enclosure.

As is obvious and clear from the plots, a more uniform illumination can be achieved by having multiple light sources spread over the roof instead of having one at the center. Further, simulations were also done for various heights of the enclosure $(h=1.5 \mathrm{~m}, 4.5 \mathrm{~m}, 6 \mathrm{~m}, 9 \mathrm{~m}$, and $18 \mathrm{~m}$ ) which shows a similar distribution of irradiance inside the enclosure.

The irradiance profiles by simulating measurements by the detectors having poor cosine responses (Detectors A, B,

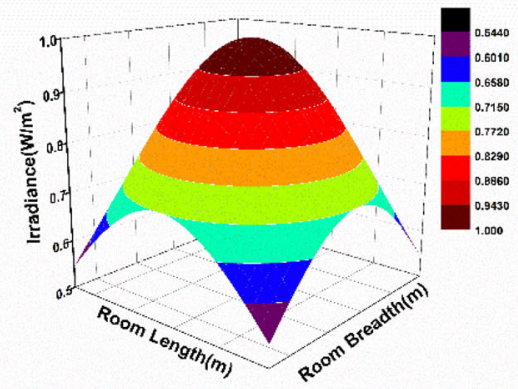

(a)

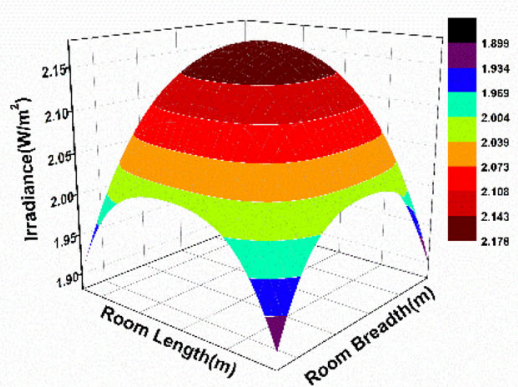

(b)

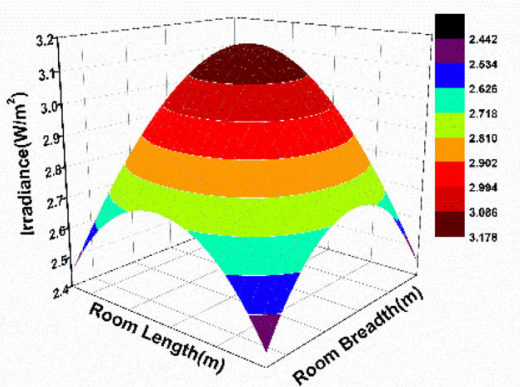

(c)

Fig. 6 Irradiance profile of the isotropic light source inside cubic enclosure under different lighting conditions: a center, $\mathbf{b}$ four corners, $\mathbf{c}$ one at the center and four at the corners 
Fig. 7 Contour plots of relative error in irradiance, measured by the four detectors, at different positions inside the cubical enclosure with one light source placed at the center of the roof
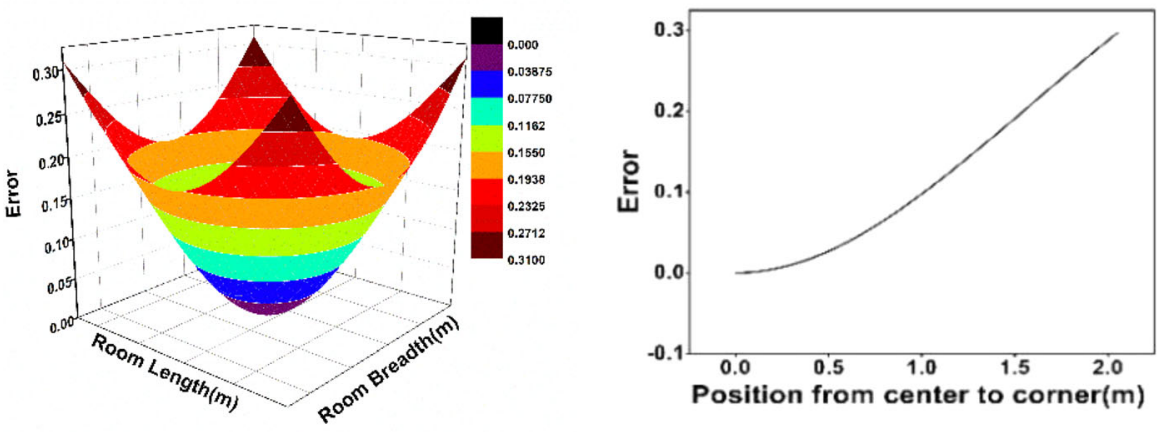

\section{Detector A}
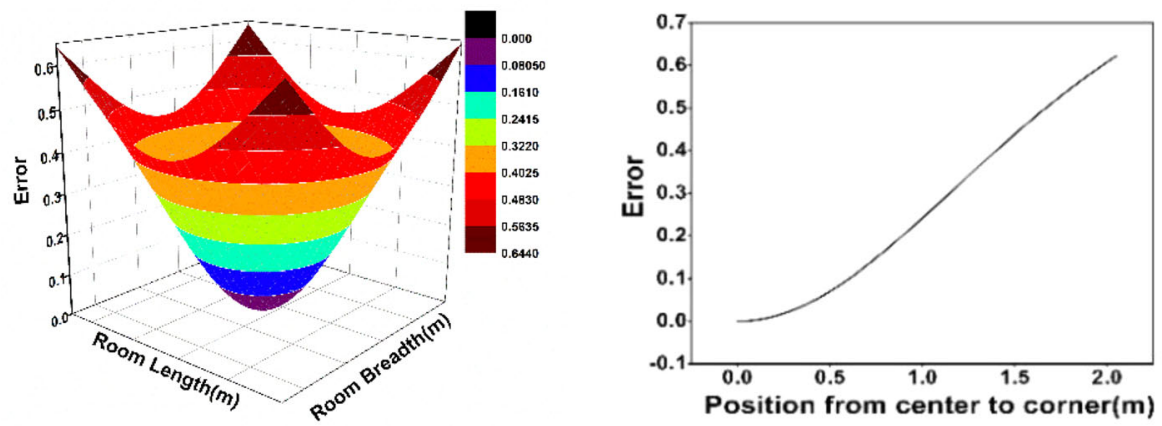

Detector B
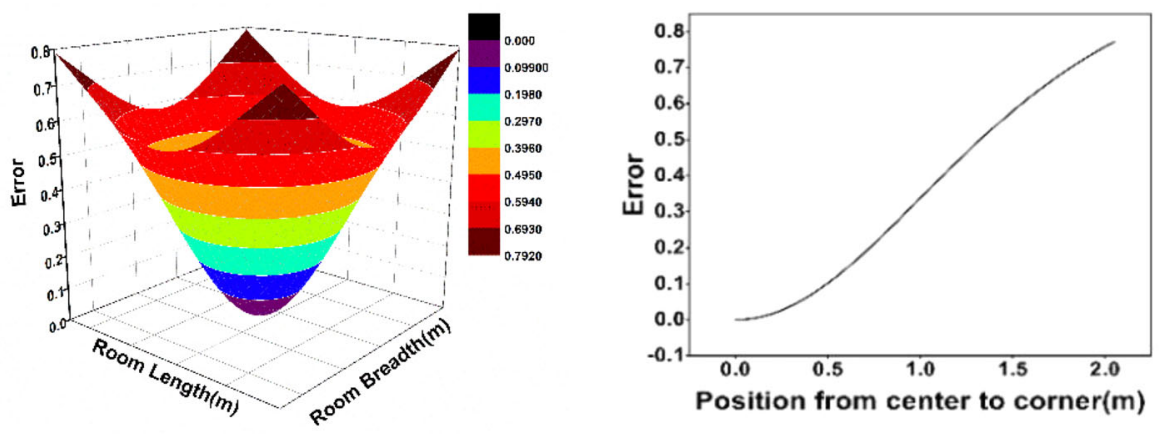

\section{Detector C}
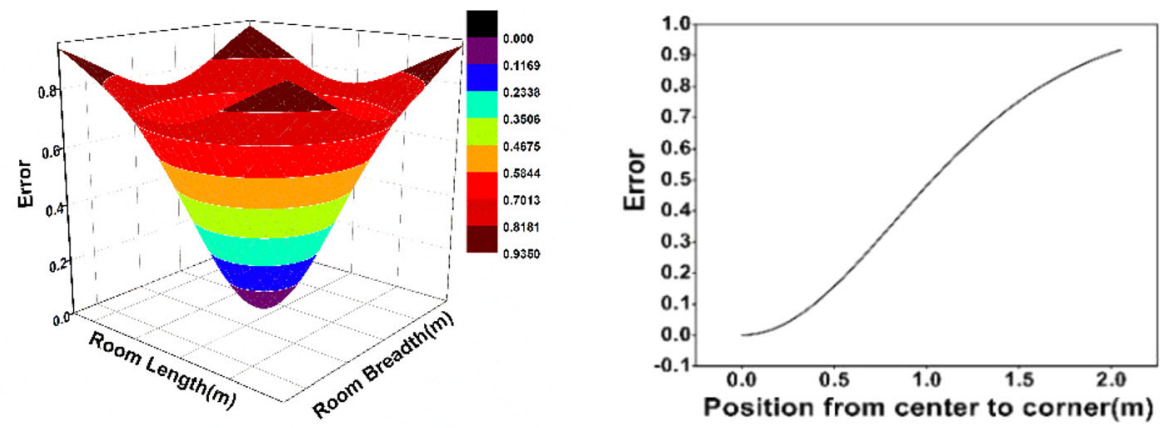

Detector D 
C, and D) are obtained under different lighting conditions. Then, relative errors were calculated for the irradiance measured by all four detectors for different lighting conditions to the irradiance measurement by the ideal detector under similar lighting conditions.

Figure 7 shows the profiles of the error in irradiance measurement made by Detectors A, B, C, and D when the light source is placed at the center of the roof. It can be observed that the errors at the center of the room are exactly zero for all the detectors. This is attributed to the fact that the light falls normally on the detectors at this position, and the calibration of the detector holds good. It is interesting to observe that in all the detectors, measurement error increases as we move away from the center toward the corners of the floor. The disparity in the errors arises from the fact that the detectors are at an oblique incidence position when placed at the corners. The same can be observed from Fig. 7 where the detectors record a lesser value of irradiance as compared to the true value on moving away from the center. Thus, the detectors provide erroneous values at the corners of the room even when they are calibrated. The maximum error recorded for the detectors varies from $31 \%$ (Detector A) to $93.5 \%$ (Detector D).

The data shown in Fig. 7 are at a fixed height of $3 \mathrm{~m}$. As the errors are expected to vary on changing the height of the enclosure, similar calculations are carried out for different heights. The maximum error occurring at the corners decreases as the height of the enclosure is increased. The variation in the recorded errors with the height of the enclosure is shown in Fig. 8. The errors first decrease sharply and then start to saturate after a particular height. This is due to the fact that on increasing the height of the

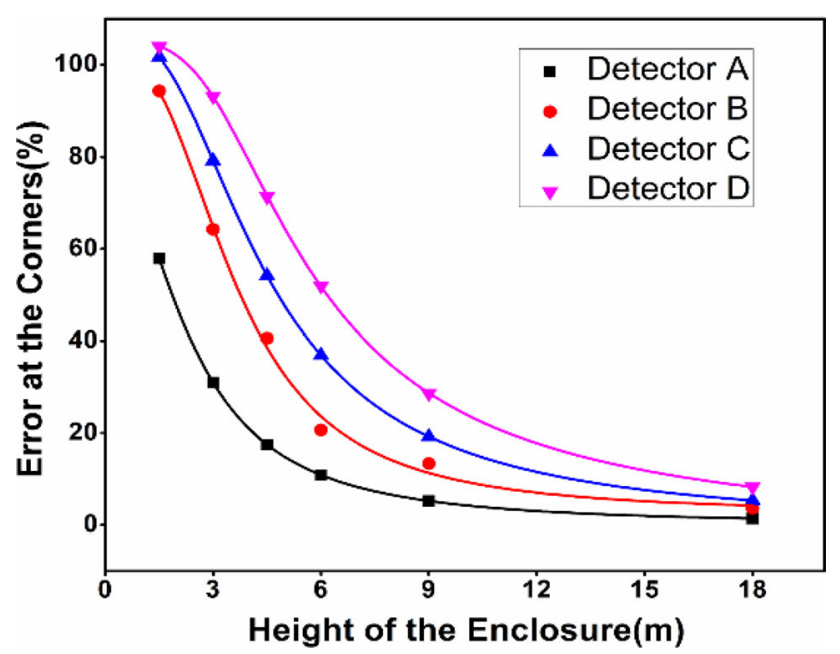

Fig. 8 Maximum error in irradiance measurement recorded by each detector at the corners of the floor for different heights of the enclosure with one light source placed at the center of the roof enclosure, the angle of incidence reduces which results in a decrease in the related measurement errors.

It is important to point out that the calculations in the present work are carried out by taking the initial size of the enclosure as $3 \mathrm{~m} \times 3 \mathrm{~m} \times 3 \mathrm{~m}$ with varying heights $1.5 \mathrm{~m}, 4.5 \mathrm{~m}, 6 \mathrm{~m}, 9 \mathrm{~m}$, and $18 \mathrm{~m}$. The errors remain similar if the ratio of the dimensions remains the same. Hence, the calculated values hold good for smaller enclosures for example a $30 \mathrm{~cm} \times 30 \mathrm{~cm} \times 30 \mathrm{~cm}$ box.

The errors in the measurement of irradiance recorded by Detectors A, B, C, and D when four isotropic light sources are placed at the corners of the enclosure of different heights are shown in Figs. 9 and 10.

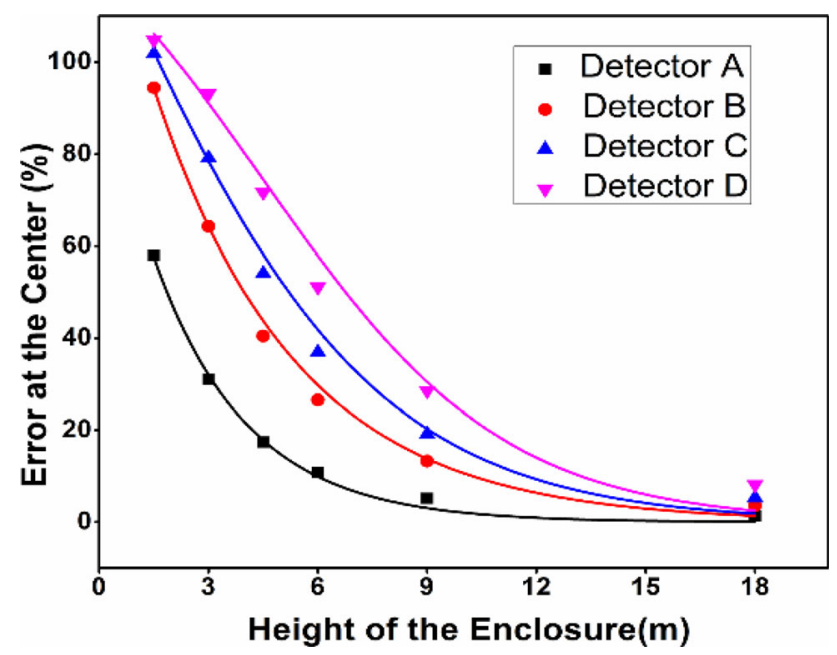

Fig. 9 Maximum error in irradiance measurement recorded by each detector at the center of the floor for different heights of the enclosure with light sources placed at the four corners of the roof

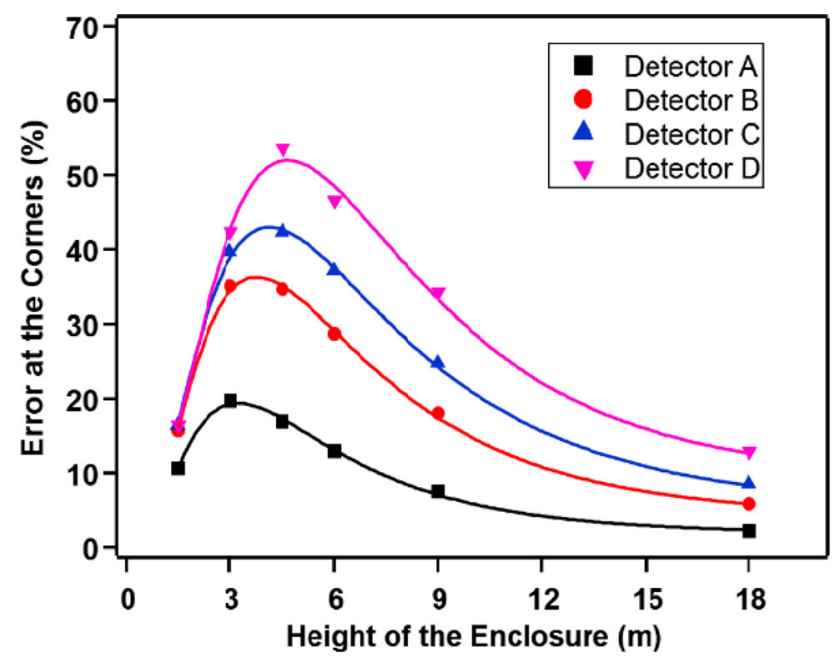

Fig. 10 Maximum error in irradiance measurement recorded by each detector at the corners of the floor for different heights of the enclosure with light sources placed at the four corners of the roof 
Theoretical simulations were done for calculating these errors at different heights: $1.5 \mathrm{~m}, 3 \mathrm{~m}, 4.5 \mathrm{~m}, 6 \mathrm{~m}, 9 \mathrm{~m}$, and $18 \mathrm{~m}$. The maximum error at the center of the floor of the enclosure (Fig. 8) is obtained for height $1.5 \mathrm{~m}$ which decreases with an increase in height. This is because the angle between the detector surface normal and light source direction progressively decreases on increasing height. The errors in the measurement of irradiance for the height of $18 \mathrm{~m}$ are $1.4 \%, 3.6 \%, 5.3 \%$, and $8.3 \%$ for Detector A, B, $\mathrm{C}$, and $\mathrm{D}$, respectively.

The errors in irradiance measurements at the corners of the floor of the enclosure show an interesting trend (Fig. 10). It first increases with height and then falls off with height. For instance, Detector A shows errors of $12.3 \%, 22.5 \%$ at heights 1.5 and $3 \mathrm{~m}$ respectively whereas this error decreases to $8.6 \%, 2.6 \%$ for height $9 \mathrm{~m}$ and $18 \mathrm{~m}$ respectively. This observation may be attributed to the fact that at smaller heights, the contribution to irradiance from the sources at the corners is quite less due to larger angles. With further increase in height, the errors decrease due to the reduction in the detector-source angle.

The theoretical simulations are also carried out to obtain the relative error in the measurements of irradiance for the setup having light sources at the center along with one at each corner of the roof (using Eq. 11).

Figure 11 shows the error recorded by each detector at the center of the floor when four light sources are placed at the corners along with one at the center of the enclosure. A similar procedure is followed to calculate errors at the corners of the floor (Fig. 12). It may be observed that in both cases errors in measurement first increase and then decrease with an increase in height of the enclosure.

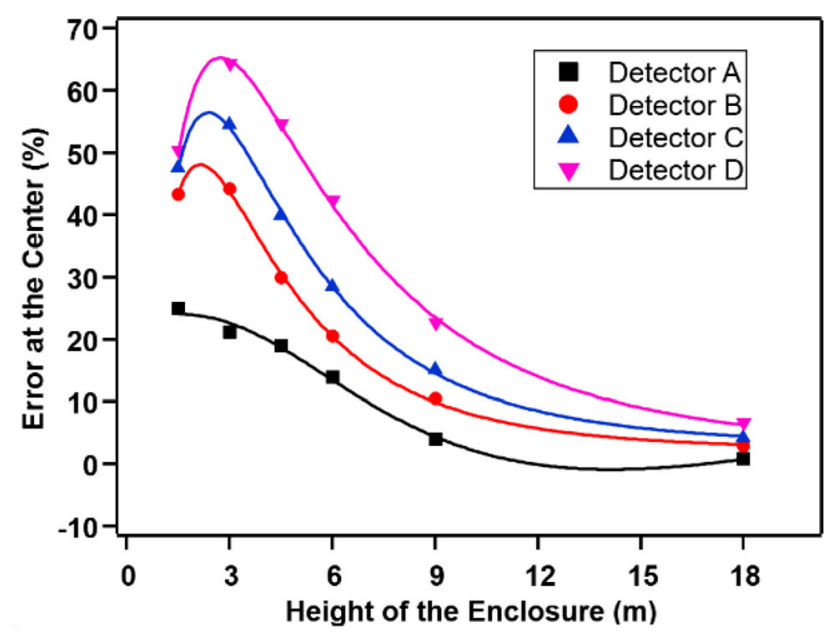

Fig. 11 Maximum error in irradiance measurement recorded by each detector at the center of the floor for different heights of the enclosure with light sources placed at four corners of the roof along with one at the center of the roof

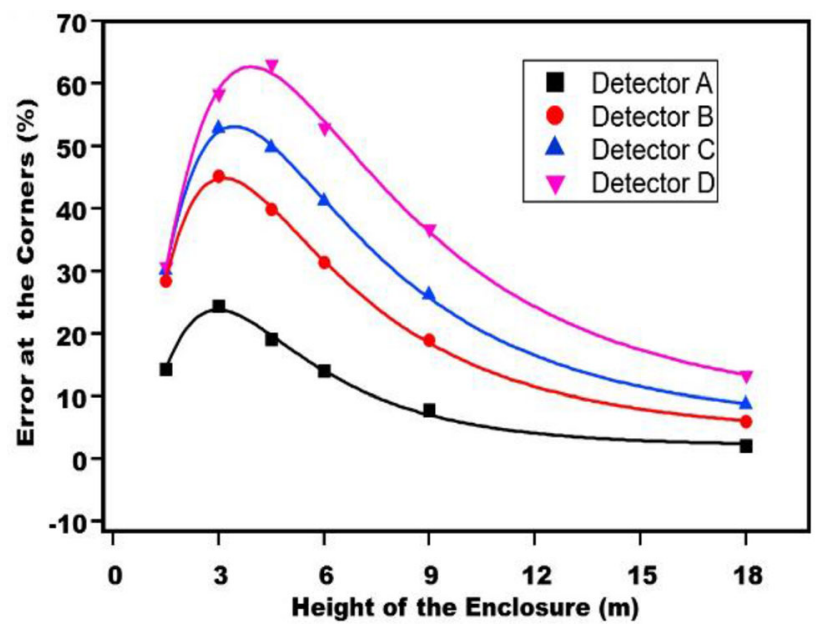

Fig. 12 Maximum error in irradiance measurement recorded by each detector at the corners of the floor for different heights of the enclosure with light sources placed at four corners of the roof along with one at the center of the roof

\section{Conclusion}

The present study highlights the importance of cosine corrections in calibrated detectors employed for irradiance measurements. Three common light source arrangements were chosen for the study, and it was observed that, in an enclosure, a more uniform illumination can be achieved by having multiple light sources installed over the roof as compared to one at the center. Four detectors (A, B, C, and D) having non-ideal cosine responses are considered to evaluate measurement error in irradiance for different lighting conditions. Error profiling shows that for one light source at the center there would be no measurement errors at the center of the floor of the enclosure and errors increase on moving away from the center to the corners of the floor. The detector $\mathrm{D}$ having $\mathrm{f}_{2}$ value of 51.66 shows an error up to $93.5 \%$ at the corners. However, the errors reduce as the height of the enclosure is increased. In the case of multiple light sources distributed over the roof, there is an error in irradiance measurement at the center which decreases on increasing height of the enclosure. At the corners, the errors in irradiance measurements first increase with height and then fall off gradually.

Apart from highlighting the importance of cosine correction of detectors, the study is also indicative of the fact that the measurement errors would maximize for a cubical enclosure as compared to a cuboidal enclosure. The facts from the study would be useful in designing the UVGI chambers where the floor width is generally larger than its height.

Acknowledgements The authors thank Director, CSIR-National Physical Laboratory, and Head, Physico-Mechanical Metrology 
Division for encouragement and support. Technical support from the members of the Optical Radiation Metrology group is acknowledged. One of the authors, Vijeta is also thankful to University Grant Commission (UGC) for providing the fellowship under the UGC-SRF scheme.

\section{References}

[1] R. Leahy, Literary illumination: the evolution of artificial light in nineteenth-century literature, University of Wales Press, (2018).

[2] S. Saha, V.K. Jaiswal, P. Sharma and D.K. Aswal, Evolution of SI base unit candela: the light perception of human eye. MAPAN-J Metrol. Soc. India, 35 (2020) 563-573.

[3] S. F. Johnston, A history of light and colour measurement, CRC Press, (2015).

[4] P. Sharma, V.K. Jaiswal and H.C. Kandpal, Ultraviolet radiation emitted by compact fluorescent Lamps. MAPAN-J Metrol. Soc. India, 24 (2009) 183-191.

[5] C. Chang, H. Liu, C. Peng, J. Shieh and C. Lan, UVR measurement of a UV germicidal lamp. Health Physics, 92 (2007) 242-250.

[6] Muhammad M.A.S Mahmoud, Economic Applications for LED Lights in Industrial Sectors, InLight-Emitting Diodes-Exciting Progress and Future Directions, IntechOpen, (2021).

[7] B.K. Yadav, D.P. Bahuguna, Jai Bhagwan, H.C. Kandpal, Illuminance meter: an important device for photometry, MAPAN-J Metrol. Soc. India, 19 (2004) 103-108.

[8] N.R. Greene, Shedding light on the candela. The Physics Teacher, 41 (2003) 409-414.

[9] M. Heßling, K. Hönes, P. Vatter and C. Lingenfelder, Ultraviolet irradiation doses for coronavirus inactivation- review and analysis of coronavirus photoinactivation studies, GMS Hygiene and Infection Control 15 (2020).

[10] K.C.P. Leung and T.C.S. Ko, Improper use of the germicidal range ultraviolet lamp for household disinfection leading to phototoxicity in COVID-19 suspects. Cornea, 40 (2021) 121-122.

[11] C.S. Heilingloh, U.W. Aufderhorst, L. Schipper, U. Dittmer, O. Witzke, D. Yang, X. Zheng, K. Sutter, M. Trilling, M. Alt, E. Steinmann and A. Krawczyk, Susceptibility of SARS- CoV-to UV irradiation. American Journal of Infection Control, 48 (2020) 1273-1275.

[12] I. Kohli, A.B. Lyons, B. Golding, S. Narla, A.E. Torres, A. Parks-Miller, D.M. Ozog, H.W. Lim and I.H. Hamzavi, UVC germicidal units: determination of dose received and parameters to be considered for N95 respirator decontamination and reuse. Photochemistry and Photobiology, 96 (2020) 1083-1087.

[13] D.R. Grimes, C. Robbins and N.J. O'Hare, Dose modeling in ultraviolet phototherapy. Medical Physics, 37 (2010) 5251-5257.
[14] I.H. Hamzavi, A.B. Lyons, I. Kohli, S. Narla, A. Parks-Miller, J.M. Gelfand, H.W. Lim and D.M. Ozog, Ultraviolet germicidal irradiation: possible method for respirator disinfection to facilitate reuse during the COVID-19 pandemic. Journal of the American Academy of Dermatology, 82 (2020) 1511-1512.

[15] K.J. Card, D. Crozier, A. Dhawan, M. Dinh, E Dolson, N. Farrokhian, V. Gopalakrishnan, E. Ho, T. Jagdish, E.S. King, and N. Krishnan, UV sterilization of personal protective equipment with idle laboratory biosafety cabinets during the Covid-19 pandemic, MedRxiv, (2020).

[16] Y. Yang, H. Zhang, V. Chan and A.C.K. Lai, Development and experimental validation of a mathematical model for the irradiance of in-duct ultraviolet germicidal lamps. Building and Environment, 152 (2019) 160-171.

[17] A.C. Parr, A national measurement system for radiometry, photometry and pyrometry based upon absolute detectors, 1421, NIST Publications (1996).

[18] P. Sharma, V.K. Jaiswal, R. Mehrotra and H.C. Kandpal, Upgradation of a spectral irradiance measurement facility at National Physical Laboratory, India. MAPAN-J Metrol. Soc. India, 25 (2010) 21-28.

[19] S.W. Brown, G.P. Eppeldauer, J.P. Rice, J. Zhang and K.R. Lykke, Spectral irradiance and radiance responsivity calibrations using uniform sources (SIRCUS) facility at NIST, InEarth Observing Systems IX. International Society for Optics and Photonics, 5542 (2004) 363-374.

[20] M. Ghazeer, A. Abdelmageed and A. Hassen, Realization of spectral irradiance responsivity at NIS-Egypt. Optik, 168 (2018) 390-395.

[21] S. Mekaoui and G. Zibordi, Cosine error for a class of hyperspectral irradiance sensors. Metrologia, 50 (2013) 187-199.

[22] K. Lakkala, A. Arola, J. Gröbner, S.F. León-Luis, A. Redondas, S. Kazadzis, T. Karppinen, J.M. Karhu, L. Egli, A. Heikkilä and T. Koskela, Performance of the FMI cosine error correction method for the Brewer spectral UV measurements. Atmospheric Measurement Techniques, 11 (2018) 5167-5180.

[23] J.J. Michalsky, L.C. Harrison and W.E. Berkheiser III., Cosine response characteristics of some radiometric and photometric sensors. Solar Energy, 54 (1995) 397-402.

[24] R. Cahuantzi and A. Buckley, Geometric optimisation of an accurate cosine correcting optic fibre coupler for solar spectral measurement. Review of Scientific Instruments, 88 (2017) 0950031.

[25] F. Vera, R. Rivera and M. Ortíz, A simple experiment to measure the inverse square law of light in daylight conditions. European Journal of Physics, 35 (2013) 0150151-0150155.

Publisher's Note Springer Nature remains neutral with regard to jurisdictional claims in published maps and institutional affiliations. 